\title{
ON THE NUMBER OF GENERATORS AND THE MODULAR GROUP-RING OF A FINITE $p$-GROUP
}

\author{
OLAF MANZ AND REINER STASZEWSKI
}

\begin{abstract}
We consider the Loewy-series $J(K P)^{i}$ of a finite p-group $P$ over a field $K$ of characteristic $p$. We point that the series is not 'monotonic' in general, but we can show that the dimensions of the Loewy-factors $J(K P)^{i} / J(K P)^{i+1}$ (except the first and the last one) are greater than or equal to the minimal number of generators of $P$.
\end{abstract}

Let $P$ be a finite $p$-group and $K$ an arbitrary field of characteristic $p$ ( $p$ a prime). In his paper [3], S. Jennings determined the dimensions of the Loewy-factors$J(K P)^{i} / J(K P)^{i+1}$ in terms of a certain central series of the group $P$. In the following, we state his result (cf. [2, Chapter VIII, $\S \S 1-2]$ ). Define the central series $P=$ $\kappa_{1}(P) \geq \kappa_{2}(P) \geq \cdots \geq \kappa_{l}(P)>\kappa_{l+1}(P)=1$ by $\kappa_{n}(P)=\left[\kappa_{n-1}(P), P\right] \kappa_{m}(P)^{p}$ (for $n>1)$, where $m$ is the least integer for which $p m \geq n$. Then $\kappa_{n}(P) / \kappa_{n+1}(P)$ is elementary abelian and we put $\operatorname{dim}_{G F(p)}\left(\kappa_{n}(P) / \kappa_{n+1}(P)\right)=d_{n}(1 \leq n \leq l)$. Further, if $J(K P)^{s}>J(K P)^{s+1}=0$, we define $\operatorname{dim}_{K}\left(J(K P)^{i} / J(K P)^{i+1}\right)=c_{i}(0 \leq$ $i \leq s)$.

TheOREM (S. Jennings [3]; CF. [2, VIII 2.10]).

$$
\prod_{n=1}^{l}\left(1+t^{n}+\cdots+t^{n(p-1)}\right)^{d_{n}}=\sum_{i=0}^{s} c_{i} t^{i}
$$

in particular, the Loewy-series is symmetric, namely $c_{i}=c_{s-i}$.

The starting-point for the following paper was a question of $\mathrm{B}$. Huppert: Is the Loewy-series even monotonic, which means $c_{i-1} \leq c_{i}$ for $1 \leq i \leq s / 2$ ? In the first section, we will show that the answer is 'no'. But we also show that for some classes of $p$-groups the Loewy-series is indeed monotonic. Let $d$ be the minimal number of generators of $P$. Then $d=d_{1}=c_{1}$, hence $J(K P) / J(K P)^{2} \cong K^{d}$. In $\S 2$, we will prove that the inequality $d \leq c_{i}$ holds for arbitrary $p$-groups $P(1 \leq i \leq s-1)$.

1. On the monotony of the Loewy-series.

1.1 CoUnTEREXAMPLE. As already mentioned, the Loewy-series is not monotonic in general.

(a) Define

$$
P=\left\{\left(\begin{array}{ccc}
1 & x & y \\
& 1 & x^{3} \\
& & 1
\end{array}\right) \mid x, y \in G F\left(3^{3}\right)\right\} .
$$

Received by the editors October 7, 1985.

1980 Mathematics Subject Classification (1985 Revision). Primary 20C05.

Key words and phrases. This paper is a contribution to the research project "Darstellungstheorie" of the DFG. 
Then $P$ is a 3-group of order $|P|=3^{6}$ and of exponent 3 ; moreover

$$
Z(P)=P^{\prime}=\left\{\left(\begin{array}{ccc}
1 & 0 & y \\
& 1 & 0 \\
& & 1
\end{array}\right) \mid y \in G F\left(3^{3}\right)\right\}
$$

(Indeed, $P$ is the universal 3-group with 3 generators, of class 2 and exponent 3.) With the notation above, we have $\kappa_{1}(P)=P, \kappa_{2}(P)=P^{\prime}, \kappa_{3}(P)=1$, hence $d_{1}=d_{2}=3$. Calculating the Jennings-polynomial yields

$$
\left(1+t+t^{2}\right)^{3}\left(1+t^{2}+t^{4}\right)^{3}=\cdots+87 t^{8}+85 t^{9}+87 t^{10}+\cdots
$$

and the Loewy-series of $K P$ has a waist.

(b) If one tries to check Huppert's question, one would start considering those groups the $\kappa$-series of which are determined in [2, VIII 2.9]. For abelian $p$-groups the conjecture is indeed true (see Proposition 1.4), but we give a counterexample for $p$-groups of maximal class. Let $P$ be a 3-group of maximal class and order $|P|=3^{5}$. Then the Jennings-polynomial is in this case

$$
\begin{array}{r}
\left(1+t+t^{2}\right)^{2}\left(1+t^{2}+t^{4}\right)\left(1+t^{3}+t^{6}\right)\left(1+t^{6}+t^{12}\right) \\
=\cdots+14 t^{12}+13 t^{13}+14 t^{14}+\cdots .
\end{array}
$$

Now we state some classes of $p$-groups the Jennings-series of which is monotonic. We call a polynomial $f=\sum_{i=0}^{m} \alpha_{i} t^{i} \in \mathbf{R}[t], \alpha_{m} \neq 0$, symmetric iff $\alpha_{i}=\alpha_{m-i}$ $(i=0,1, \ldots, m) ; f$ is called monotonic iff $\alpha_{i-1} \leq \alpha_{i}(1 \leq i \leq m / 2)$.

1.2 LEMMA. Let $f=\sum_{i=0}^{m} \alpha_{i} t^{i} \in \mathbf{R}[t]$ be symmetric and monotonic. Then so is $f \cdot\left(\sum_{j=0}^{n} t^{j}\right)$.

ProOF. Of course, $\sum_{j=0}^{m+n} \beta_{j} t^{j}:=f \cdot\left(\sum_{j=0}^{n} t^{j}\right)$ is symmetric. We put $\alpha_{i}=0$ for negative integers $i$, hence $\beta_{j}=\sum_{k=0}^{n} \alpha_{j-k}$. We have to show that $\beta_{j}-\beta_{j-1}=$ $\alpha_{j}-\alpha_{j-1-n}$ is nonnegative for all $0 \leq j \leq(m+n) / 2$. This is clear if $j \leq m / 2$, because $f$ is monotonic. Suppose therefore that $m / 2<j \leq(m+n) / 2$. The symmetry of $f$ shows $\alpha_{j}=\alpha_{m-j}$, where $m-j \leq m / 2$. Now $j \leq(m+n) / 2$ yields $j-(n+1) \leq m-j$, which finally means $\alpha_{j-(n+1)} \leq \alpha_{m-j}=\alpha_{j}$ and $\beta_{j}-\beta_{j-1} \geq 0$ is shown.

1.3 LEMMA (HUPPERT, BLACKBURN [2, VIII 2.9]). Let $P$ be abelian. Then $d_{n}=0$ if $n$ is not a power of $p$ and $d_{1} \geq d_{p} \geq d_{p^{2}} \geq \cdots$.

1.4 Proposition. If $P$ is abelian, then the Loewy-series of $K P$ is monotonic.

ProOF. Jennings' Theorem and Lemma 1.3 imply

$$
\begin{aligned}
\sum_{i=0}^{s} c_{i} t^{i}= & \prod_{k=1}^{r}\left(1+t^{p^{k}}+\cdots+t^{p^{k}(p-1)}\right)^{d_{p^{k}}} \\
= & \left(1+t+t^{2}+\cdots+t^{p^{r+1}-1}\right)^{d_{p^{r}}} \\
& \cdot\left(1+t+t^{2}+\cdots+t^{p^{r}-1}\right)^{d_{p^{r-1}}-d_{p^{r}}} \cdots \\
& \cdot\left(1+t+t^{2}+\cdots+t^{p^{2}-1}\right)^{d_{p}-d_{p^{2}}} \\
& \cdot\left(1+t+t^{2}+\cdots+t^{p-1}\right)^{d_{1}-d_{p}} .
\end{aligned}
$$


Now Lemma 1.2 at once yields the result by induction.

We prove Proposition 1.4 for a larger class of $p$-groups. Therefore, we need a lemma, analogous to 1.3 .

1.5 LEMMA. Let $P$ be a regular p-group (in the sense of $P$. Hall) and let $P^{\prime} \leq$ $P^{p}$. Then we have $\kappa_{n}(P)=P^{p^{i}}$ for $p^{i-1}<n \leq p^{i}$.

PROOF. Induction on $n$. By definition, $\kappa_{1}(P)=P$. Let $n>1$, then $\kappa_{n}(P)=$ $\left[\kappa_{n-1}(P), P\right] \kappa_{m}(P)^{p}$, where $m$ denotes the minimal natural number with $p m \geq n$. Choose $i>0$, such that $p^{i-1}<n \leq p^{i}$; hence $p^{i-2}<m \leq p^{i-1}$.

Case 1. $p^{i-1}+1<n \leq p^{i}$. This yields by induction

$$
\kappa_{n}(P)=\left[P^{p^{i}}, P\right]\left(P^{p^{i-1}}\right)^{p}=P^{p^{i}} .
$$

Case 2. $n=p^{i-1}+1$. Hence

$$
\begin{aligned}
\kappa_{p^{i-1}+1}(P) & =\left[\kappa_{p^{i-1}}(P), P\right] \kappa_{p^{i-2}+1}(P)^{p} \\
& =\left[P^{p^{i-1}}, P\right]\left(P^{p^{i-1}}\right)^{p} \quad \text { (by induction) } \\
& =\left(P^{\prime}\right)^{p^{i-1}} P^{p^{i}} \quad(\text { by }[1, \text { III } 10.8]) \\
& =P^{p^{i}} \quad\left(\text { as } P^{\prime} \leq P^{p}\right) . \quad \square
\end{aligned}
$$

1.6 Proposition. Let $P$ be a regular $p$-group and $P^{\prime} \leq P^{p}$. Then the Loewyseries of $K P$ is monotonic.

PROOF. By Lemma $1.5, d_{n}=0$ if $n$ is not a power of $p$. On the other hand, $d_{1} \geq d_{p} \geq d_{p^{2}} \geq \cdots$ (by $[1$, III 10.7]), and the proof of Proposition 1.4 runs through.

We close $\S 1$ considering another class of frequently appearing $p$-groups.

1.7 PROPOSITION. If $P$ is an extra-special p-group, then the Loewy-series is monotonic.

ProOF. Let $|P|=p^{2 m+1}$. Clearly, $\kappa_{2}(P)=Z(P)=\phi(P)$.

Case 1. $\operatorname{Exp}(P)=p^{2}$. By definition of the $\kappa$-series, we obtain $d_{1}=2 m, d_{2}=$ $\cdots=d_{p-1}=0, d_{p}=1, d_{i}=0(i>p)$, and the proof of Proposition 1.4 yields the result.

Case 2. $\operatorname{Exp}(P)=p$. Then $d_{1}=2 m, d_{2}=1$ and $d_{i}=0(i>2)$. By Lemma 1.2 it is obviously sufficient to consider $|P|=p^{3}$. Now the Jennings-polynomial is

$$
\sum_{i=0}^{s} c_{i} t^{i}=\left(1+t+\cdots+t^{p-1}\right)^{2}\left(1+t^{2}+\cdots+t^{2(p-1)}\right) .
$$

If $\sum_{i=0}^{s / 2} \alpha_{i} t^{i}=\left(1+t+\cdots+t^{p-1}\right)^{2}$, then $\alpha_{i}=i+1$ for $0 \leq i \leq p-1$ and $\alpha_{2(p-1)-i}=\alpha_{i}$. We put again $\alpha_{i}=0$ for negative integers $i$. As $c_{j}=\sum_{i=0}^{p-1} \alpha_{j-2 i}$, we get $c_{j}-c_{j-1}=\sum_{i=0}^{p-1}\left(\alpha_{j-2 i}-\alpha_{j-1-2 i}\right)$. If $j \leq p-1$, then clearly $c_{j}-c_{j-1} \geq 0$ and we are done. Suppose therefore $p-1<j \leq 2(p-1)$. We count the number of summands $\alpha_{j-2 i}-\alpha_{j-1-2 i}$ which are 1 (denoted by $n_{1}$ ) and which are -1 (denoted by $n_{-1}$ ).

(a) We have $\alpha_{j-2 i}-\alpha_{j-1-2 i}=1$ iff $0 \leq j-2 i \leq p-1$, hence $n_{1} \geq(p-1) / 2$.

(b) On the other hand, $\alpha_{j-2 i}-\alpha_{j-1-2 i}=-1$ iff $p-1<j-2 i \leq 2(p-1)$, hence $n_{-1} \leq(j-p) / 2+1$. 
By (a) and (b),

$$
\begin{aligned}
c_{j}-c_{j-1} & =n_{1}-n_{-1} \geq(p-1) / 2-((j-p) / 2+1) \\
& \geq(p-1) / 2-((2(p-1)-p) / 2+1) \geq-1 / 2 .
\end{aligned}
$$

As $c_{j}-c_{j-1}$ is an integer, we have $c_{j}-c_{j-1} \geq 0$ and the proof is complete.

2. A lower bound for the orders of the Loewy-factors. Clearly,

$$
K P / J(K P) \cong J(K P)^{s} \cong K .
$$

Furthermore, $J(K P) / J(K P)^{2} \cong K^{d}$, where $d$ denotes the minimal number of generators of $P$. In this section, we show that $d$ is a lower bound for the dimensions of the Loewy-factors $J(K P)^{i} / J(K P)^{i+1}$ for $1 \leq i \leq s-1$. This generalizes a result of B. Külshammer [4], who proved the following: If $J(K P)^{i} / J(K P)^{i+1} \cong K$ for some $i(1 \leq i \leq s-1)$, then $P$ is cyclic and all Loewy-factors are isomorphic to $K$.

We start stating some consequences of the definition of the central series $\kappa_{n}(P)$.

2.1 LEMMA. If $d_{n+1}=d_{n+2}=\cdots=d_{n p}=0$, then $d_{i}=0$ for all $i>n p$.

PROOF. Suppose not. We choose a minimal index $i$ such that $\kappa_{i}(P)>\kappa_{i+1}(P)$ and $i>n p$. Let further $m$ be the minimal natural number such that $p m \geq i+1$. By the definition of the $\kappa$-series, we have $\kappa_{i+1}(P)=\left[\kappa_{i}(P), P\right] \kappa_{m}(P)^{p}$.

We claim that $n+1 \leq m \leq i$ : Since $m p \geq i+1>n p$, we conclude $m \geq n+1$. On the other hand, the minimal choice of $m$ yields $i+1>p(m-1)$, hence $m \leq i$. As $\kappa_{i}(P)=\kappa_{n p}(P)$, the equation $\kappa_{i+1}(P)=\left[\kappa_{n p}(P), P\right] \kappa_{n+1}(P)^{p}$ holds. But $n+1$ is the minimal natural number such that $p(n+1) \geq n p+1$, hence $\kappa_{i+1}(P)=$ $\kappa_{n p+1}(P)=\kappa_{i}(P)$, a contradiction.

To simplify our notation, we denote those $d_{i}$ which are different from 0 by $d_{m_{1}}$ (= $\left.d_{1}=d\right), d_{m_{2}}, \ldots, d_{m_{k}}, \ldots, d_{m_{e}}$. In these terms, the assertion of Lemma 2.1 runs as follows: $m_{k} \leq p m_{k-1}(2 \leq k \leq e)$. In addition, we define $s_{k}=(p-1) \sum_{i=1}^{k} m_{i} d_{m_{i}}$; by Jennings' Theorem $s_{e}=s$.

2.2 LEMMA. With the notations above, we have

$$
s_{k} \geq p m_{k}+(p-1)(d-1)-1 .
$$

ProOF. We use induçtion on $k$.

$$
\begin{aligned}
k=1: \quad s_{1} & =(p-1) m_{1} d_{m_{1}}=p m_{1} d-d=p m_{1}+p(d-1)-d \\
& =p m_{1}+(p-1)(d-1)-1 . \\
k>1: \quad s_{k} & =s_{k-1}+(p-1) m_{k} d_{m_{k}} \\
& \geq p m_{k-1}+(p-1)(d-1)-1+(p-1) m_{k} d_{m_{k}} \quad \text { (by induction) } \\
& \geq p m_{k}+(p-1)(d-1)-1+\left(p m_{k-1}-m_{k}\right) \quad\left(\text { as } d_{m_{k}} \geq 1\right) \\
& \geq p m_{k}+(p-1)(d-1)-1 \quad(\text { by Lemma 2.1). } \quad \square
\end{aligned}
$$

As an easy consequence of Lemmata 2.1 and 2.2 we obtain

2.3 LEMMA. $m_{k} \leq s_{k-1}-(p-1)(d-1)+1$.

We are now able to prove the main result of this section. 
2.4 THEOREM. Let $d$ as before be the minimal number of generators of the p-group $P$ and $c_{n}(0 \leq n \leq s)$ the dimensions of $J(K P)^{n} / J(K P)^{n+1}$, the Loewyfactors. Then

$$
c_{n} \geq d(1 \leq n \leq s-1) .
$$

Proof. If $d=1$, then $P$ is cyclic and all $c_{n}=1$. Therefore, we may assume $d \geq 2$. We put

$$
\begin{aligned}
f_{k} & =\prod_{j=1}^{k}\left(1+t^{m_{j}}+\cdots+t^{(p-1) m_{j}}\right)^{d_{m_{j}}} \\
& =: \sum_{n=0}^{s_{k}} c_{n}^{(k)} t^{n} \quad \text { for } k=1, \ldots, e .
\end{aligned}
$$

In particular, $c_{n}^{(e)}=c_{n}$. Of course, $c_{1}^{(k)}=d_{m_{1}}=d$ for all $k$. We show by induction on $k: c_{n}^{(k)} \geq d$ for $1 \leq n \leq s_{k}-1$.

$$
k=1: \quad f_{1}=\left(1+t^{m_{1}}+\cdots+t^{(p-1) m_{1}}\right)^{d_{m_{1}}}=\left(1+t+\cdots+t^{(p-1)}\right)^{d} .
$$

By Lemma 1.1, $f_{1}$ is symmetric and monotonic. As $c_{1}^{(1)}=d$, the assertion holds.

$$
k>1: \quad f_{k}=f_{k-1}\left(1+t^{m_{k}}+\cdots+t^{(p-1) m_{k}}\right)^{d_{m_{k}}}
$$

and the induction yields $c_{n}^{(k-1)} \geq d$ for $1 \leq n \leq s_{k-1}-1$.

Let $1 \leq l \leq s_{k}-1$. Then

$$
c_{l}^{(k)} \geq \sum_{i=0}^{(p-1) d_{m_{k}}} c_{l-i m_{k}}^{(k-1)}
$$

where the $c_{r}^{(k-1)}$ are understood to be 0 for negative indices $r$. Clearly, 1$(p-1) d_{m_{k}} m_{k} \leq s_{k-1}-1$, as $s_{k}-1-(p-1) d_{m_{k}} m_{k}=s_{k-1}-1$. Moreover, Lemma 2.3 yields $m_{k} \leq s_{k-1}$.

(1) Suppose at first $m_{k} \leq s_{k-1}-1$. Then $M:=\left\{l, l-m_{k}, l-2 m_{k}, \ldots, l-\right.$ $\left.\left[(p-1) d_{m_{k}}\right] m_{k}\right\} \cap\left\{1,2, \ldots, s_{k-1}-1\right\} \neq \varnothing$. We take $n \in M$ and our induction hypothesis shows $c_{l}^{(k)} \geq c_{n}^{(k-1)} \geq d$.

(2) It remains to consider the case $m_{k}=s_{k-1}$. If even in this case $M \neq 0$, we can proceed as in (1). Finally, let $M=\varnothing$; then there is an index $i$ such that $l-i m_{k}=0$ and $l-(i-1) m_{k}=s_{k-1}$, consequently $c_{l}^{(k)} \geq c_{0}^{(k-1)}+c_{s_{k-1}}^{(k-1)}=2$. On the other hand, the assumption (2) and Lemma 2.3 show $p=d=2$, hence $c_{l}^{(k)} \geq 2=d$.

As already mentioned, the Loewy-series remains stationary from the beginning (which means of course all Loewy-factors are isomorphic to $K$ ) if and only if $P$ is cyclic. In view of Theorem 2.4 , it seems to be of interest to determine those groups $P$ for which the Loewy-series is stationary from the second up to the penultimate factor.

2.5 PROPOSITION. The Loewy-series of $K P$ is stationary from the second up to the penultimate factor (which means $d=c_{1}=c_{2}=\cdots=c_{s-1}$ ) if and only if one of the following cases occurs:

(1) $P$ is cyclic,

(2) $p=2$ and $P \cong \mathbf{Z}_{2} \times \mathbf{Z}_{2} \times \mathbf{Z}_{2}$,

(3) $p=2$ and $P$ has a cyclic normal subgroup of index 2 . 
PROOF. " $\Leftarrow$ " If $P$ is cyclic, then all Loewy-factors are isomorphic to $K$. If $P \cong \mathbf{Z}_{2} \times \mathbf{Z}_{2} \times \mathbf{Z}_{2}$, then the Jennings-polynomial is $(1+t)^{3}=1+3 t+3 t^{2}+t^{3}$.

So, let $P$ have a cyclic normal subgroup $C$ of index 2 and $p=2$. It is easy to see that $\kappa_{j}(P)=C^{2^{i}}$ for $2^{i-1}<j \leq 2^{i}$ and $j \geq 2$. Hence we obtain the Jennings-polynomial of $P$ (for some integer $n$ )

$$
\begin{aligned}
& (1+t)^{2}\left(1+t^{2}\right)\left(1+t^{4}\right) \cdots\left(1+t^{2^{n}}\right) \\
& =(1+t) \cdot \sum_{i=0}^{2^{n+1}-1} t^{i}=1+2 \sum_{i=1}^{2^{n+1}-1} t^{i}+t^{2^{n+1}} .
\end{aligned}
$$

Therefore, all but the first and the last Loewy-factors have dimension 2 .

" $\Rightarrow$ " If $s=1$ or $s=2$, then $P \cong \mathbf{Z}_{2}, \mathbf{Z}_{3}$ or $\mathbf{Z}_{2} \times \mathbf{Z}_{2}$. Hence, we may assume $s \geq 3$. To calculate $c_{1}$ and $c_{2}$ it is obviously enough to consider the polynomial

$$
\left(1+t+t^{2}+\cdots+t^{p-1}\right)^{d} \cdot\left(1+t^{2}+\cdots+t^{2(p-1)}\right)^{d_{2}} .
$$

(Note that $d_{2}$ may be 0 .) Of course, we have $c_{1}=d$.

(a) Suppose first $p>2$. Then $c_{2}=d+\left(\begin{array}{l}d \\ 2\end{array}\right)+d_{2}$ and $c_{2}=c_{1}$ yields $d_{2}=0$ and $d=1$, hence $P$ is cyclic.

(b) In the case $p=2$ we obtain $c_{2}=\left(\begin{array}{l}d \\ 2\end{array}\right)+d_{2}$ and $c_{2}=c_{1}$ yields $d^{2}-3 d+2 d_{2}=0$, consequently $d \leq 3$.

(1) If $d=1$, then $P$ is cyclic.

(2) Secondly, $d=3$ forces $d_{2}=0$. This implies $d_{i}=0$ for all $i \geq 2$ (by Lemma 2.1), hence $P$ is elementary abelian and $P \cong \mathbf{Z}_{2} \times \mathbf{Z}_{2} \times \mathbf{Z}_{2}$.

(3) Finally, suppose $d=2$, hence $d_{2}=1$ and $2=d=c_{1}=\cdots=c_{s-1}$. We put as in the proof of Theorem 2.4

$$
f_{k}=\prod_{j=1}^{k}\left(1+t^{m_{j}}\right)^{d_{m_{j}}}=\sum_{n=0}^{s_{k}} c_{n}^{(k)} t^{n} \quad(k=1, \ldots, e) .
$$

Trivially, $c_{1}^{(k)}=\cdots=c_{s_{k-1}}^{(k)}=2$ for all $k$.

( $\alpha$ ) We claim $d_{m_{k}}=1$ for all $k \geq 2$. Suppose not. Hence

$$
\begin{aligned}
f_{k}=f_{k-1} \cdot\left(1+t^{m_{k}}\right)^{d_{m_{k}}=} & \left(1+2 t+\cdots+2 t^{s_{k-1}-1}+t^{s_{k-1}}\right) \\
& \cdot\left(1+d_{m_{k}} t^{m_{k}}+\cdots+t^{d_{m_{k}} m_{k}}\right)
\end{aligned}
$$

and the coefficient of $t^{m_{k}+1}$ is at least $2 \cdot d_{m_{k}}>2$, a contradiction.

( $\beta$ ) We now claim that $P / \kappa_{m_{k}+1}(P)$ has a cyclic normal subgroup of index 2 $(k=1, \ldots, e)$ : We use induction on $k$. If $k=1$, then $P / \kappa_{2}(P)$ is of type $(2,2)$. In case of $k=2$, we have $m_{2}=2\left(\right.$ as $\left.d_{2}=1\right)$ and $P / \kappa_{3}(P)$ has order 8 and 2 generators. Let $k>2$. By $(\alpha), \kappa_{m_{k}}(P) / \kappa_{m_{k}+1}(P)$ has order 2. Let $Z=\kappa_{m_{k}}(P)=$ $\kappa_{m_{k-1}+1}(P)$ and write $\bar{P}=P / \kappa_{m_{k}+1}(P)$. By the induction hypothesis, $\bar{P} / \bar{Z}$ has a normal cyclic subgroup $\langle x \bar{Z}\rangle$ of index 2 . If $\bar{Z}=\langle z\rangle$ and $z$ is a power of $x$, certainly $\langle x\rangle$ is the desired subgroup of $\bar{P}$. In the other case, $\langle x\rangle \times\langle z\rangle \cong \mathbf{Z}_{2^{\alpha}} \times \mathbf{Z}_{2}$ has index 2 in $\bar{P}$. But now it is clear that $z \notin \overline{\kappa_{3}(P)}$, a contradiction. As $\kappa_{m_{e}+1}(P)=1,(\beta)$ completes the proof.

2.6 REMARK. The 2-groups with a cyclic normal subgroup of index 2 are completely described in Huppert $[\mathbf{1}, \mathrm{I}, 14.9]$. The list consists of abelian groups, 
the quaternion, dihedral and semidihedral groups; furthermore the groups $P=$ $\left\langle g, h / g^{2^{n}}=h^{2}=1, g^{h}=g^{1+2^{n-1}}\right\rangle$ belong to the list.

\section{REFERENCES}

1. B. Huppert, Endliche Gruppen I, Springer, Berlin, 1979.

2. B. Huppert and N. Blackburn, Finite groups II, Springer, Berlin, 1982.

3. S. Jennings, The structure of the group ring of a p-group over a modular field, Trans. Amer. Math. Soc. 50 (1941), 175-185.

4. B. Külshammer, Symmetric local algebras and small blocks of finite groups, J. Algebra 88 (1984), 190-195.

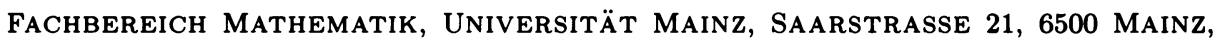
WEST GERMANY 